\title{
SUBACUTE PLAGUE IN MAN DUE TO GROUND SQUIRREL INFECTION.*
}

\author{
GEORGE W. M CCOY, \\ Passed Assistant Surgeon, U. S. Public Health and Marine Hospital Service, \\ AND \\ WILLIAM B. WHERRY, \\ Acting Assistant Surgeon, U. S. Public Health and Marine Hospital Service.
}

The subject of this report was a boy (J. M.) born in California of Portuguese parents. His age was $\mathrm{I} 3$ years and he was rather more than ordinarily well developed physically for his age. There was nothing in the previous history, medical or otherwise, having any bearing on the present illness, with the exception of the fact that, according to his own statement, he had been shooting ground squirrels in Alameda County, California, about a week before he was taken sick. According to the father's statement the boy had been out squirrel hunting just five days prior to his becoming ill. While on this hunting trip he shot four or five squirrels which he brought home with him. He stated that he had seen sick squirrels in the same vicinity, but did not shoot nor handle any of them. ${ }^{x}$ This boy had not been more than a few miles from his home in his life and indeed had never seen a trolley car until he was brought to the hospital in Oakland. There is no evidence that he was in any place where he might have been infected with plague except on this hunting trip. There had been no known previous case of plague in man in California for nearly a year, and none in rodents, except ground squirrels, for about seven months.

The boy became sick on Tuesday, July 27 , with headache and violent vomiting. The vomiting was almost continuous for about 24 hours. The next day he was seen by a physician and was found to have a temperature of $104^{\circ} \mathrm{F}$. On Thursday, 48 hours after the beginning of the illness, the glands in the left axilla were found to be enlarged and tender. The patient was taken to a hospital in Oakland,

* Received for publication, October 26 , I

"Since the above was written a plague infected squirrel has been shot in the immediate vicinity of where the boy had been hunting.-G. W. M., W. B. W. 
California, where the glands in the left axilla were excised and found to be "broken down." They were not submitted to microscopical or bacteriological examination. It was distinctly stated that no pus was present.

The temperature did not fall materially after the glands were removed, but on the following day was about $104^{\circ} \mathrm{F}$. On the second day after the operation it ranged between $99^{\circ}$ and Io2 $2^{\circ}$. On Sunday, the third day after the operation (the fifth day of the illness), it was ${ }_{102}{ }^{\circ} \mathrm{F}$. On this day all of the superficial glands were markedly enlarged and tender, those of the neck and groins being especially prominent. Suspicion that the boy might be suffering from plague was now aroused. Information of the case was communicated to Surgeon Rupert Blue, commanding anti-plague operations in California, and by his instructions we investigated the case. At this time the boy's expression was anxious, his face flushed, his breathing rather rapid, pulse about $I 20$, temperature $102^{\circ} \mathrm{F}$, and he had the general appearance of being very sick. The cervical and inguinal glands were enlarged, fused together, and tender. The clinical picture was one characteristic of plague, and with the previous history did not leave one in any serious doubt as to the diagnosis. The left inguinal gland was excised with aseptic precautions and the tissues submitted to us and to Drs. Nusbaumer and Archibald for microscopical and bacteriological examination. Smears from the inguinal gland which had been excised on this day for diagnostic purposes showed numerous bacilli, which in shape, size, and staining reaction were identical with $B$. pestis.

Cultures made from these glands on agar gave a translucent sticky growth quite characteristic of $B$. pestis, and sub-cultures on salt agar gave well marked involution forms after 18 hours. Stalactites were developed in the broth culture and the reactions on the other media were those of $B$. pestis. Two guinea-pigs were inoculated, one by the cutaneous method; this animal died on the seventh day with characteristic lesions of plague and from its tissues $B$. pestis was recovered in pure culture. The other guinea-pig was inoculated subcutaneously, and died on the fifth day. Both had characteristic lesions of plague and from each one pure cultures of $B$. pestis were recovered. A white rat was inoculated by the subcutaneous 
method with a piece of the gland and died on the third day with characteristic lesions of plague and a pure culture of B. pestis was isolated from its liver. Two white rats were vaccinated, that is, inoculated by the cutaneous method; one died on the fourth day, presenting the usual lesion of plague in rats. B. pestis was isolated from its liver. The other rat was killed on the I 2 th day and was found to be normal.

The boy was isolated at the Alameda County Infirmary during the next ten days; the temperature ranged from $100^{\circ} \mathrm{F}$. to $103^{\circ} \mathrm{F}$. He was given 40 c.c. of Yersin's anti-plague serum daily, but no very material change took place in his condition until the roth day of the illness when a pustular eruption appeared which was uniformly distributed over the body. The pustules varied in diameter from $2 \mathrm{~mm}$. to Io $\mathrm{mm}$. and each one was at first surrounded by a red areola. Pus taken from one of these lesions on the 12 th day of the disease showed no bacteria in smear preparations but a guinea-pig inoculated subcutaneously with the pus died on the third day with characteristic lesions of early plague, and a pure culture of the plague bacillus was isolated from its liver. Another guinea-pig vaccinated with the same pus was killed on the seventh day and found to be entirely free from any lesions. As early as the fifth day of the illness the sputum was slightly tinged with blood, but it bore no resemblance to the sputum in pneumonic plague. This tinging with blood persisted only a day or two. On the I2th day of the illness a rather irregular area of dulness was found at the base of the left lung by the attending physician. On the I3th day marked exophthalmos appeared but without increase of ocular tension. Small tubercle-like bodies appeared on the iris at the margin of the left pupil. The exophthalmos became extreme, the breathing rapid, the pulse fast, and the boy died on the 16 th day of the illness.

\section{POST MORTEM FINDINGS.}

The body is somewhat emaciated; the skin of a brownish and slightly yellowish color; post-mortem lividity of the dependent portions of the trunk and neck is fairly well marked; sclerae intensely icteric; the anterior edges of the cornea of both eyes show a few irregular petechiae and in the ocular conjunctiva above the left eye 
are two submucous hemorrhages about $3 \mathrm{~mm}$. in diameter; the anterior chamber of the right eye is somewhat cloudy; the pupils are irregular and in the iris near the edge of the left pupil are a few yellowish nodules $0.5^{-1} \mathrm{~mm}$. in diameter. There is a bloody discharge from the right ear and a purulent one from the left.

There is a marked swelling on both sides of the neck, extending from below the ears to the region of the cervical glands; on section these glands are seen to be almost completely broken down into a pinkish purulent material.

There is an operation wound in the left axilla and another in the left femoral region; the right axillary glands are just palpable; there is a prominent bubo in the right femoral region and on section the right and left femoral and inguinal glands are seen to have undergone a necrosis similar to that of the cervical glands. The epitrochlear glands are palpable.

The muscles are dark red on section. Both pleural cavities are about half full of a clear amber colored fluid. The lungs are voluminous. Scattered irregularly throughout the substance of all the lobes of both lungs are many irregular tumor-like nodules which are of a light yellowish color. These vary in size from $2 \mathrm{~mm}$. to $5 \mathrm{~cm}$. in diameter. They are raised slightly above the pleural surface, feel hard on pressure, and many of them are surrounded by a zone of congestion. On section they are seen to be composed of a firm pinkish gray tissue surrounding a central area of softening There are a few subpleural petechiae on the lower lobes of the lungs (see Fig. I).

The pericardial sack contains a small amount of clear yellowish fluid. The heart is of about normal size. The auricular epicardium is of a yellowish color. The aortic, mitral, and tricuspid valves appear normal. A yellowish fibrinous clot is loosely adherent to the walls of the right ventricle.

There is no fluid in the peritoneal cavity. The appendix is normal. The visceral peritoneum shows nothing abnormal. Intestines not opened. The spleen is considerably enlarged and of a fairly firm texture; its capsule is smooth and of a light purple color, mottled with areas of deep purple and these darker areas are seen on section to extend into its substance. 
674 George W. McCoy and William B. Wherry

The right and left kidneys are alike, they are intensely congested, their capsules strip readily; each shows half a dozen subcapsular abscesses, 2-4 $\mathrm{mm}$. in diameter; on section the cortex appears swollen and its markings are indistinct; section through some of the abscesses shows that some of them are surrounded by an area of intensely congested tissue. The adrenals are both enlarged and show marked congestion on section.

The liver is congested; just beneath the capsule in the center of the right lobe is an abscess about $3 \mathrm{~mm}$. in diameter; the cut surface has a nutmeg appearance.

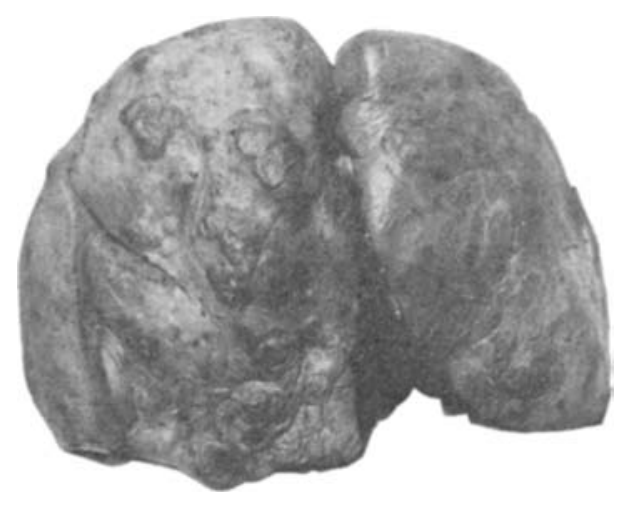

FIG. I. - Human lungs from fatal case of squirrel plague

Anatomic Diagnosis.-Bubonic, lobular pneumonic, and pyemic plague; hydrothorax; passive congestion of the liver; acute nephritis, sub-acute splenitis; multiple abscesses in the regional glands, lungs, liver, and kidneys.

Smears made from one of the lung nodules showed a few typical bipolar bacilli and a few involution forms bearing a strong resemblance to those seen in the early growth of the plague bacillus on salt agar. We might state here that the only other occasions on which we have seen these involution forms from animal tissues have been in a few cases of squirrel plague. We have never seen them in human plague, rat plague, nor in the guinea-pig.

The bacteriological examination of one of the lung nodules taken at the post mortem examination yielded the following results:

A pure culture of $B$. pestis was isolated from one of the lung 
nodules by culture; a guinea-pig inoculated by the cutaneous method with a piece of a lung nodule died on the fifth day with typical lesions of plague and a culture of the plague bacillus was isolated from its liver. Another guinea-pig was inoculated from the inguinal bubo. This pig was killed on the I4th day and found to be entirely normal.

Surgeon Rupert Blue has suggested to us that, in his experience, cases of plague in man, in which the infection was derived from squirrels, are apt to be less virulent than where the infection has been derived presumably from rats. The present case might, perhaps, lend some support to this view. We have seen but one other case of plague which was undoubtedly due to squirrel infection. In that case the patient recovered after a long and severe illness and after having developed a general plague septicemia. Two cases of plague occurred a year ago in Contra Costa County, California, both of which possibly derived their infection from squirrels, and these cases died of acute plague on the third and fourth day of the disease respectively. In these four cases, two of which certainly and two very likely derived their infection from squirrels, the bubo was situated in the axillary region in each case.

In the majority of cases of human plague due to rat infection the bubo is in the inguinal region.

We would call special attention to the following points:

I. This is the first case of undoubted squirrel plague in man which has come to autopsy in America.

2. The lesions were similar to those of sub acute plague in guineapigs, rats, and squirrels. We have never seen lesions of the same nature in any other case of human plague; in fact, without any knowledge of the previous history of the case one would scarcely have suspected plague infection at autopsy.

We wish to acknowledge our indebtedness to Surgeon Rupert Blue, Dr. W. A. Clark, superintendent of the Alameda County Infirmary, and Dr. H. E. Morrison, of Niles, for data used in preparing the present report. 